\title{
http://bjas.journals.ekb.eg \\ Recent Updates and Guidelines in Anesthetic Management of Obstetric Emergencies
}

M.A.Hamoda, M.H.Abdel Rahman, M.A.Khashaba and M.A.S.abdel-Aziz

Anesthesia and Intensive Care Dept., Faculty of Medicine, Benha Univ., Benha, Egypt

E-Mail : Doc.azzazy18@gmail.com

\begin{abstract}
The pregnant mother is subjected to major changes in her anatomy and physiology to can feed and accommodate the growing baby. Such changes initiate subsequently after gestation have an influence on body organs. It is essential to discriminate between normal physiological changes and pathological changes. The anesthetist is considered a substantial part in the management of risky pregnancies, and must be a member of the multidisciplinary team who is assigned to concern for seriously ill parturient. Considerations must be taken to physiological modifications during treatment. The aim of this work is to highlight the risk factors and etiology of obstetric emergencies and how to manage them based on recent updates and guidelines.
\end{abstract}

\section{Introduction}

Two persons in obstetrics are concerned to rather than one, a parturient and a fetus. The managing of one patient intensely influences the managing of the other. At times the choice has to be made to care for one person at the cost of the other; i.e., mother comes first before the second [baby] may be feasible or not [1].

During conception, the parturient experiences noteworthy physiological and anatomical changes to feeding, and nestle the developing baby. These changes start after inception of pregnancy and affect all organ system in the body. It is essential to discriminate between normal physiological changes and disease pathology [2].

Numerous anesthesiologists dread to deal with obstetric emergencies because they signify one of the crucial challenges in their daily work. Obstetric emergencies can be lethal to the mother and her baby, hemorrhage can be disastrous and unavoidable so it is important for immediate intervention, obstetric emergencies consisting of obstetric hemorrhage, fetal compromise, and specific circumstances, for instance pregnancyinduced hypertension [PIH], difficult airway, and maternal cardiac arrest [3].

The anesthetist is considered a substantial part in the management of risky pregnancies, and must be a component of the multidisciplinary team who is assigned to concern for seriously ill parturient. Anesthetists must be qualified in advanced life support and how to resuscitate and to provide required safe anesthesia and pain management [4] .

\section{Obstetric hemorrhage}

Obstetric hemorrhage is still the most significant world's cause of maternal dying and extreme morbidity. $30 \%$ of entirely maternal death accounts for peripartum hemorrhage in developing countries as Asia and Africa [5].

severe peripartum bleeding is contributed to serious morbidity as infertility, Sheehan's syndrome , respiratory failure, renal failure and defects in coagulation [6].

\subsection{Common causes of obstetric hemorrhage 2.1.1 Antepartum Hemorrhage}

Placenta previa, abruptio placentae and uterine rupture.

\subsubsection{Postpartum Hemorrhage}

Uterine atony, Genital trauma and Placenta accrete.

\subsubsection{Coagulation Disorders}

Hemophilia A, Hemophilia B, von Willebrand disease, Glanzmann thrombasthenia and BernardSoulier syndrome [7] .

\subsection{Antepartum obstetric hemorrhage Placenta previa}

1 in 200 parturiencies complaining of placenta previa and considered by the presence of the placenta covering the endocervical os [8].

Risk factors involving a previous placenta previa and previous uterine surgery are common. The placenta separation from the uterine decidua leads to considerable bleeding with uteroplacental insufficiency. Painless vaginal bleeding remains the classical symptom with or without abdominal pain [9].

\section{Uterine rupture}

defined as a complete thickness detachment of the wall of uterus and the covering serosa, happens in nearly 1 in 100 pregnant happens with a previous uterine surgery [10].

Increase incidence of rupture of uterus with factors interfering the amalgamation of the wall of uterus for example; grand multiparity, fetal malpresentation, and induction of labor with high oxytocin doses [11].

\subsection{Postpartum obstetric hemorrhage}

Postpartum hemorrhage is described in the first day of giving birth as losing of $500 \mathrm{ml}$ of blood or more from the female genital tract. Massive postpartum hemorrhage is considered as $1000 \mathrm{ml}$ blood loss or more which may be minor [500-1000 $\mathrm{ml}$ ] or major more than $1000 \mathrm{ml}$ [12]. 
Uterine atony is the reason for almost $80 \%$ of postpartum hemorrhages [6].

\subsection{Management of obstetric hemorrhage}

Most mortality related to obstetric hemorrhage is avoidable, with "substandard care" participating to nearly $70 \%$ of maternal death. Unfavorable consequences have been identified to several preventable factors, including absence of blood product availability, underestimation of blood loss inadequate communication among team members and late invasive interventions [13].

\section{Hypertensive disorders of pregnancy \\ 3.1 Choice of anesthesia}

\subsubsection{Regional anesthesia}

Spinal or epidural anesthesia can be adjusted securely when the patient is aware, with no seizure and with stable vital signs without any signs of high intracranial pressure. Maternal and neonatal results when comparing epidural and general anesthesia for cesarean section in aware women with eclampsia do not differ [14].

A good option is spinal anesthesia with small dose of heavy marcain and fentanyl. Spinal anesthesia is more preferable than GA in eclamptics [15].

During cesarean section inducted by spinal anesthesia, there is less incidence of hypotension for preeclamptic than healthy pregnant patients. Combination heavy marcain $7.5 \mathrm{mg}$ with fentanyl $25 \mu \mathrm{g}$ give sufficient anesthesia [16].

Contraindications to spinal anesthesia involve patient refusal, disseminated intravascular coagulation and abruptio placentae. With regard to spinal anesthesia inducted to patients with aspirin, the American society for regional anesthesia has revealed that a low-dose aspirin treatment is not a contraindication for regional anesthesia. Regional anesthesia is safe with platelet count of more than $75 \times 10^{3} \mathrm{mg} / \mu \mathrm{L}$. Platelet count $<50000 \mathrm{mg} / \mu \mathrm{L}$ is commonly considered a contraindication. Between the range $50-75$ thousands $\mathrm{mg} / \mu \mathrm{L}$ an patient assessment [take in consideration patient risk factors and coagulation profile] is essential [17].

\section{Difficult airway}

Obstetric patients are at higher risk of failure of tracheal intubation during general anesthesia due to a number of distinctive clinical and human factors. In spite of progresses in airway equipment and techniques, the rate of failed and difficult obstetric intubation has not altered for more than 30 years and still greater portion than in the non obstetric patients [18].

Difficult intubation has been stated to happen in 1 in 21 obstetric intubations with comparison to 1 in 50 non obstetric intubations [19].

\subsection{Pulmonary aspiration risk reduction}

Methods to prevent and decrease the occurrence of pulmonary aspiration of gastric contents are corner stone in management of obstetric general anesthesia. Detection of gastric contents and risk of regurgitation can be assessed by using ultrasound, it has lately been reported, but still more investigations are requisite [20].

\subsection{Intrauterine fetal resuscitation}

Intrauterine resuscitation ought to be started instantly, and methods to improve blood flow of uterus for example left lateral tilt and using tocolysis, are possibly more efficient than maternal oxygen supplementation, however in cases of placental insufficiency it is useful. Reevaluation of surgery urgency must be done before proceeding [21].

\subsection{Plan with team}

The World Health Organization surgical checklist has to be applied prior to every step [22].

This checklist may be modified locally for caesarean section, in some medical units are using certain anesthetic checklist is developed additionally [23].

The anesthetist has to be told by the obstetrician about all medical details related to the case and its progression. A second anesthetist must be available if needed and waiting his attendance for induction of anesthesia if it is possible. Standardization of airway equipment is extremely recommended and. there should be emergency airway crash cart and the anesthetist must be aware of it contents and it ought to be checked regularly and every once in a while. [24]

The predominant indications to prefer general anesthesia is maternal compromise with failed to resuscitate, and acute fetal distress [particularly if awake intubation or a rapid spinal anesthesia is unfeasible]. Presence of periglottic airway edema and advancing airway obstruction are fair enough indication to wake the pregnant up in the existence of supraglottic airway devices or facemask.

\subsection{Rapid sequence induction}

All members of the team have to keep calm during preparation and anesthesia induction to decrease distraction and to confirm that all staff is acquainted with the developments of events.

\subsection{Patient Positioning}

Optimal position is crucial preceding to of general anesthesia induction in all patients and particularly in pregnant patients [25].

\subsection{Cricoid pressure}

There is debate for using of cricoid pressure. The society of difficult airway guidelines advise application of cricoid pressure within rapid sequence induction. If a supraglottic airway device is necessary after tracheal intubation fails, we 
should stop cricoid pressure temporary and insert laryngeal mask then apply cricoid pressure again [24].

\subsection{Deliver adequate doses of induction agent and neuromuscular blocking drug}

Propofol is the best choice with strongly recommended for causes that include easy recovery, fewer drug faults and also anesthetist more familiar with it. Propofol also suppresses airway reflexes more than thiopental [26]. The Fifth National Audit Project of the Royal College of Anesthetists and the AAGBI [NAP5] reported a higher rate of incidence of awareness in obstetrics, and underlined inadequately small doses of thiopental sodium less than $4 \mathrm{mg}$ per $\mathrm{kg}$ as one of the possible causes [27].

\subsection{Face Mask Ventilation Prior to Tracheal Intubation}

Mask ventilation before intubation has mostly been eluded during rapid sequence induction as fear of gastric distension and high incidence of regurgitation, but with properly applied cricoid pressure this should not happen [28].

\subsection{First intubation attempt}

Anesthetists need to be aware with the advantage and limitations of the direct laryngoscopes. A short-handled Macintosh laryngoscope was previously the preference in the UK for tracheal intubation of pregnant patients. Nowadays videolaryngoscopes generally give a better view for the glottis than direct laryngoscopes [29].

\subsection{Second intubation attempt}

If the first attempt for intubation fails, the most qualified anesthetist should make the second attempt using as appropriate alternative equipment [30].

Only an experienced anesthetist will make a third attempt for intubation. To order to prevent awareness, an extra dose of intravenous anesthetic has to be given [27].

\subsection{Verify tracheal intubation}

A monitoring capnographic trace is the most dependable method for approving tracheal intubation. Severe bronchospasm or tracheal tube blockage may infrequently cause absent ventilation with a flat capnograph pattern despite a properly placed endotracheal tube [31].

\subsubsection{Front-of-neck procedure}

The recommendations for performance a frontof-neck procedure are evolving frequently with respect to equipment, technique and also human factors. A technique of small bore cannula has increased the rate of failure, particularly in patients with morbid obese. A surgical airway gives a ultimate access airway with a better rate of success [32].

\subsubsection{Debriefing and follow-up}

After an anticipated or unanticipated difficult airway, debriefing of duties is an important chance for team to reveal their achievement. Successful debriefing is finished by recognizing features of proper performance, points that need to be improved and recommendations of what better be achieved in a different manner in the future. [32]

Awareness is more common during difficult intubation, and feedback should be asked about this in the follow-up visit [33].

\section{Fetal compromise}

Non reassuring fetal status is an expression to characterize assumed fetal hypoxia and is intended to substitute the more universal term "fetal distress". Fetal distress, is progressive fetal hypoxia with/without acidemia secondary to insufficient fetal oxygenation, is a term that is used to imply changes in fetal heart patterns, fetal growth restriction, decreased fetal movement and presence of meconium stained fluid [34].

Non reassuring fetal status is not a disease, but an indicator of an implicit illness that results in transient or persistent oxygen lack to the baby that results in metabolic acidosis and fetal hypoxia. Meanwhile fetal oxygenation is reliant on maternal oxygenation and disorders of maternal oxygenation, placental perfusion, uterine blood supply gas transport between the baby and placenta surely result in non-reassuring fetal status and fetal hypoxia [35].

\section{Maternal cardiac arrest}

One of clinical scenarios that has the most challenging managment in medicine is cardiac arrest. Though most structures of resuscitation of arrested pregnant are like to adult resuscitation, its components has different parts and considerations. The most observable difference is that two patients exist, the mother and the baby. To order to prevent and treat cardiac arrest during pregnancy, anesthetists should have a comprehensive understanding of maternal mortality. Maternal death is described as a woman's death during pregnancy and up to 42 days after delivery or abortion, backing up the etiology of death is relevant to or exacerbated by parturiency or pregnancy management. Current data from the US Nationwide Inpatient Sample reports that cardiac arrest happen in 1:12 000 delivery admissions [36]. Internationally, 800 maternal deaths happen every day [5].

\subsection{Etiology}

Hemorrhage, heart failure, amniotic fluid embolism and sepsis were the most widespread causes of maternal cardiac arrest. $38 \%$ of them for 
hemorrhage, and $15 \%$ for heart failure and acute myocardial infarction [36] .

\subsection{Management of cardiac arrest}

First of all, declaration of code blue. The maternal code blue team is multidisciplinary including obstetricians, perinatologists, anesthesiologists, intesivists, cardiothoracic surgeons, neonatologists, and nurses. The team leader must be in position, and a duty has to be assigned to each members of the team. Remember that chest compressions have to be applied to several rotating sections [every $2 \mathrm{~min}$ ] for more effective CPR

\section{Amniotic Fluid Embolism}

Is an unusual condition however possibly a disastrous complication of pregnancy that happens during or immediately after labor [37].

Recent studies approximate the rate of incidence about 2.2 to 7.7 per 100,000 deliveries [38].

About $70 \%$ of them occur during giving birth, $19 \%$ in cesarean section, and $11 \%$ after labor in the recent postpartum [39].

Pathophysiology : Amniotic fluid itself contains many substances like vasoactive, tumor necrosis factor alpha, interleukins, platelet activating factor, tissue factor, leukotrienes C4 and D4, arachidonic acid, endothelin and others factors when emit into the maternal circulation resulting in vasoconstriction, bronchoconstriction and coagulopathy [40].

\section{Status asthmaticus}

Bronchial Asthma is one of the common chronic medical disease affecting pregnancy [41].

The rate of incidence of asthma reported in pregnant women sway between $3 \%$ and $12 \%$ [41].

Some patients may advance refractory status asthmaticus which characterized by a severe exacerbation of asthma, with no response to the usual treatment like bronchodilator and corticosteroid therapy and requires admitting to intensive care unit and perhaps need mechanical ventilation [42].

The effect of asthma on maternal and fetal with asthma aggravations include: low birth weight, preterm labor and delivery, preeclampsia, hyperemesis gravidarum, oligohydramnios, congenital malformations, gestational diabetes, high risk of cesarean section, and perinatal mortality. These complications were thought to associate with recurrent hypoxia particularly in poorly controlled asthma [43].

\section{Summary}

Obstetric bleeding remains the world's priming reason of maternal mortality. If obstetric hemorrhage is expected to occur as in patients with uterine scar or low placenta, we should insert two large intravenous cannulae for rapid infusion, cross matched blood, blood warmer, invasive monitors, cell salvage, and interventional radiological procedures should be taken into account. If placenta accrete is diagnosed during antenatal period, it will be correlated with lower maternal and neonatal morbidity including lower hemorrhage at labour and transfusion of fewer units of blood products.

Bonnar outlines a five step management plan to manage massive obstetric hemorrhage in unanticipated hemorrhage which consists of Organization of multidisciplinary team, Restoring of blood volume, Correcting of defective coagulation, Evaluation of treatment response and treatment the causes of bleeding.

Hypertensive disorders of pregnancy, like preeclampsia, affect about $10 \%$ of pregnancies all over the world, being one of the leading reasons of maternal morbidity and mortality. Obstetric patients are at higher risk of failure of tracheal intubation during general anesthesia because of a number of distinctive clinical and individual factors. The first obstetric guidelines are set for management of difficult and failed tracheal intubation during general anesthesia have been established by The Obstetric Anaesthetists' Association [OAA] and Difficult Airway Society [DAS]. They consist of 4 algorithms and 2 tables.

Cardiac arrest in pregnancy considered one of the utmost challenging in clinical cases and scenarios in medicine. There is similarity in most structures of cardiopulmonary resuscitation between arrested pregnant and adult resuscitation, however there are various different sides and considerations.

Amniotic fluid embolism is fundamentally a clinical diagnosis and you need to exclude other causes first so it is counted as a diagnosis of exclusion, and no definite test to affirm the diagnosis. The classical triad of respiratory distress, cardiovascular insufficiency and coagulopathy make it simple to diagnose the classical type. Sudden cardiorespiratory compromise in postpartum patient or in any pregnant must be considered amniotic fluid embolism until proven otherwise.

\section{References}

[1] M. Daniel Avery, Obstetric emergencies, American Journal of Clinical Medicine, Spring, Vol. 6, PP. 42, 2009.

[2] G. Locktich, Clinical biochemistry of pregnancy. Crit Rev Clin Lab Sci, Vol. 34, PP. 67-139, 1997.

[3] Chantal Crochetie`re, Obstetric Emergencies. Current Anaesthesia and Critical Care, Vol. 11, PP. 80-85, 2000.

[4] Felicity Plaat, Role of the anaesthetist in obstetric. critical care, Best Practice \& Research Clinical Obstetrics and Gynaecology, Vol. 22, PP. 917-935, 2008. 
[5] I. Say, D. Chou and A. Gemmill, Global causes of maternal death a WHO systematic analysis. Lancet Glob Health, Vol. 2, PP. e323-33, 2014.

[6] BT. Bateman, MF. Berman and LE. Riley, The epidemiology of postpartum hemorrhage in a large, nationwide sample of deliveries. Anesth Analg, Vol. 110, PP. 1368-73, 2010.

[7] Baird, Identification and Management of Obstetric Hemorrhage. Anesthesiology Clinics, Vol. 35, PP. 15-34, 2017.

[8] JA. Cresswell, C. Ronsmans and C. Calvert, Prevalence of placenta praevia by world region a systematic review and meta-analysis. Trop Med Int Health, Vol. 18, PP. 712-24, 2013.

[9] RM. Silver, Abnormal placentation placenta previa, vasa previa, and placenta accreta.Obstet Gynecol, Vol. 126, PP. 654-68, 2015.

[10] M. Kaczmarczyk, P. Sparen and P. Terry, Risk factors for uterine rupture and neonatal consequences of uterine rupture a populationbased study of successive pregnancies in sweden. BJOG, Vol. 114, PP. 1208-14, 2007.

[11] A. Vedat , B. Hasan and A. Ismail, Rupture of the uterus in labor a review of 150 cases. Isr $\mathbf{J}$ Med Sci, Vol. 29, PP. 639-43, 1993.

[12] G. Carroli, C. Cuesta, E. Abalos and AM. Gulmezoglu, Epidemiology of postpartum haemorrhage A systematic review. Best Pract Res Clin Obstet Gynaecol, Vol. 22, PP. 9991012, 2008.

[13] T. Girard, M. Mortl and D. Schlembach, New approaches to obstetric hemorrhage the postpartum hemorrhage consensus algorithm. Curr Opin Anaesthesiol, Vol. 27, PP. 267-74, 2014.

[14] J. Moodley, G. Jjuuko and C. Rout, Epidural compared with general anaesthesia for caesarean delivery in conscious women with eclampsia. Br J ObstetGynaecol, Vol. 108, PP. 378-82, 2001.

[15] M. Razzaque, K. Rahman and R. Sashidharan, Spinal is safer than GA for LSCS in eclamptics abstract. Anesthesiology, Vol. 94, PP. A34, 2001.

[16] AG. Aya, R. Mangin, N. Vialles, Patients with Severe Preeclampsia experience less hypotension during spinal anesthesia for elective cesarean delivery than healthy parturients A Prospective cohort comparison. AnesthAnalg, Vol. 97, PP. 867-72, 2003.

[17] TT. Horlocker, DJ. Wedel, JC. Rowlingson, Regional anesthesia in the patient receiving antithrombotic or thrombolytic therapy, American Society of Regional Anesthesia and Pain Medicine Evidence-Based Guidelines. 3rd ed. RegAnesth Pain Med, Vol. 35, PP. 64-101, 2010.

[18] SM. Kinsella, AL. Winton, MC. Mushambi, Failed tracheal intubation during obstetric general anaesthesia a literature review. Int J Obstet Anesth, Vol. 24, PP. 356-374, 2015.

[19] DM. McKeen, RB. George, Difficult and failed intubation Incident rates and maternal, obstetrical, and anesthetic predictors. Can J Anesth, Vol. 58, PP. 514-524, 2011.

[20] P. Van de Putte and A. Perlas, Ultrasound assessment of gastric content and volume. Br J Anaesth, Vol. 59, 11312-22, 2014.

[21] JA. Thurlow and SM. Kinsella, Intrauterine resuscitation active management of fetal distress. Int J Obstet Anesth;Vol.11, PP. 10516, 2002.

[22] AB. Haynes, TG. Weiser, WR. Berry, A surgical safety checklist to reduce morbidity and mortality in a global population. New England Journal of Medicine, Vol. 360, PP. 491-9, 2009.

[23] MD. Wittenberg, DJA. Vaughan and DN. Lucas A novel airway checklist for obstetric general anaesthesia. International Journal of Obstetric Anesthesia, Vol. 22, PP. 264-5, 2013.

[24] MC. Mushambi, SM. Kinsella, AL. Winton, Obstetric Anaesthetists' Association and Difficult Airway Society guidelines for the management of difficult and failed tracheal intubation in obstetrics. Anaesthesia, Vol. 70, PP. 1286-1306, 2015.

[25] JS. Collins, JM. Lemmens, JB. Brodsky, Laryngoscopy and morbid obesity a comparison of the "sniff", and "ramped" positions. Obes Surg, Vol. 14, PP. 1171-1175, 2004.

[26] JP. Stone, LB. Fenner and TR Christmas, The preparation and storage of anaesthetic drugs for obstetric emergencies a survey of UK practice. International Journal of Obstetric Anesthesia, Vol. 18, PP. 242-8, 2009.

[27] JJ. Pandit, J. Andrade, DG. Bogod, 5th National Audit Project NAP5 on accidental awareness during general anaesthesia summary of main findings and risk factors. Anaesthesia, Vol. 69, PP. 1089-101, 2014.

[28] JP. Brown and GC. Werrett, Bag-mask ventilation in rapid sequence induction. A survey of current practice among members of the UK Difficult Airway Society. European Journal of Anaesthesiology, Vol. 32, PP. 446$8,2015$.

[29] H. Swales, M. Mushambi and A. Winton, Management of failed intubation and difficult airways in UK obstetric units an OAA survey. International Journal of Obstetric Anesthesia, Vol. 23, PP. S19, 2014.

[30] IP. Latto, M. Stacey, J. Mecklenburgh, Survey of the use of the gum elastic bougie in clinical practice. Anaesthesia, Vol. 57, PP. 379-84, 2002.

[31] R. Haldar, S. Samanta and H. Bhagat, Unexpected tracheal tube blockage from a 
semi-dissolved misoprostol tablet. International Journal of Obstetric Anesthesia, Vol. 22, PP. 267-8, 2013.

[32] T. Cook, N. Woodall and C. Frerk, Major complications of airway management in the UK results of the Fourth National Audit Project of the Royal College of Anaesthetists and the Difficult Airway Society. Part 1 anaesthesia Br J Anaesth. May, Vol. 106, PP. 617-31, 2011.

[33] CL. Errando, JC. Sigl, M. Robles, Awareness with recall during general anaesthesia a prospective observational evaluation of 4001 patients. British Journal of Anaesthesia, Vol. 101, PP. 178-85, 2008.

[34] J.T, Parer and E.G. Livingston, What is fetal distress?.Am J Obstet Gynecol. Jun, Vol. 162, PP. 1421-5; discussion 1425-7, 1990.

[35] C.B. Martin, Normal fetal physiology and behavior, and adaptive responses with hypoxemia.Semin Perinatol. Aug, Vol. 32, PP. 239-42, 2008.

[36] JM. Mhyre, LC. Tsen, S. Einav, Cardiac arrest during hospitalization for delivery in the United States, 1998-2011.Anesthesiology, Vol. 120, PP. 810-818, 2014.

[37] P. Sultan, K. Seligman and B. Carvalho, Amniotic fluid embolism update and review.
Curr Opin Anaesthesiol, Vol. 29, PP. 288-296, 2016.

[38] CL. Roberts, CS. Algert, M. Knight, Amniotic fluid embolism in an Australian populationbased cohort. BJOG, Vol. 117, PP. 1417-1421, 2010.

[39] G. Pantaleo, N. Luigi, T. Federica, Amniotic fluid embolism Review. Curr Pharm Biotechnol, Vol. 14, PP. 1163-7, 2014.

[40] E. Turillazzi, M. Neri, S. Bello, Amniotic fluid embolism Moving diagnosis through the time. From the mechanical pulmonary vascular occlusion until an immuno -inflammatory pathogenesis? Curr Pharm Biotechnol, Vol. 14, PP. 1179-88, 2014.

[41] V. Murphy, V. Clifton and P. Gibson, Asthma exacerbations during pregnancy incidence and association with adverse pregnancy outcomes. Thorax, Vol. 61, PP. 169-176, 2006.

[42] JA. Namazy and M. Schatz, Pregnancy and asthma recent developments. Curr Opin Pulm Med, Vol. 11, PP. 56-60, 2005.

[43] D. Elsayegh and JM. Shapiro, Management of the obstetric patient with status asthmaticus. J Intensive Care Med. Nov-Dec, Vol. 23, PP. 396-402, 2008. 